\title{
Encoding of local and global cues in domestic dogs' spatial working memory
}

\author{
Sylvain Fiset ${ }^{*}$ Nathalie Malenfant
}

Université de Moncton, Campus d'Edmundston, Edmundston, Canada;

${ }^{*}$ Corresponding Author: sylvain.fiset@umoncton.ca

Received 16 May 2013; revised 17 June 2013; accepted 29 June 2013

Copyright (C) 2013 Sylvain Fiset, Nathalie Malenfant. This is an open access article distributed under the Creative Commons Attribution License, which permits unrestricted use, distribution, and reproduction in any medium, provided the original work is properly cited.

\begin{abstract}
The current study investigated whether domestic dogs encode local and/or global cues in spatial working memory. Seven dogs were trained to use a source of allocentric information (local and/or global cues) to locate an attractive object they saw move and disappear behind one of the three opaque boxes arrayed in front of them. To do so, after the disappearance of the target object and out of the dogs' knowledge, all sources of allocentric information were simultaneously shifted to a new spatial position and the dogs were forced to follow a U-shaped pathway leading to the hiding box. Out of the seven dogs that were trained in the detour problem, only three dogs learned to use the cues that were moved from trial to trial. On tests, local (boxes and experimenter) and/or global cues (walls of the room) were systematically and drastically shifted to a new position in the testing chamber. Although they easily succeeded the control trials, the three dogs failed to use a specific source of allocentric information when local and global cues were put in conflict. In discussion, we explore several hypotheses to explain why dogs have difficulties to use allocentric cues to locate a hidden object in a detour problem and why they do not differentiate the local and global cues in this particular experimental setting.
\end{abstract}

Keywords: Local and Global Cues; Spatial Working Memory; Domestic Dogs; Allocentric and Egocentric Cues; Object Permanence

\section{INTRODUCTION}

In most animals, the ability to solve spatial problems is essential for survival. For example, remembering the location of a food cache or traveling from and to nest (or home) are some of the basic spatial problems encountered by animals on a daily basis. Over the years, comparative researchers have extensively investigated spatial cognition and have reported the use of several cues and mechanisms in the spatial domain by animals (for a review see [1-4]).

Although animals can encode and use multiple sources of spatial information to remember and locate a spatial position, these sources can be roughly grouped into two categories: egocentric and allocentric cues [5]. Egocentric cues refer to the information provided by the spatial coordinates of the animal and its own movements through space [6]. Two forms of egocentric information have been identified. In its simplest form, which is called linear egocentric information, the animal plans and maintains from its starting position a direct path towards a specific location. A most complex form of egocentric information refers to dead reckoning (also known as path integration). This latter is used by animals to keep track of their displacements in space by encoding inertial information such as direction, distance and speed. However, egocentric spatial information is inflexible: if the relationship between the animal's position and the target location is broken (e.g. by the introduction of an obstacle that forces the animal to deviate from its planned trajectory), egocentric information would orient the animal towards an incorrect location. As for it, allocentric spatial information refers to the relationships between a specific location and the objects surrounding it [6-9]. Examples of allocentric cues are trees or rocks that can be used by the animal to record a location. By contrast to egocentric information, allocentric cues provides a flexible source of information because an animal can reach its target location by following different routes and reorient itself by using one or several familiar objects in the environment. Allocentric information is therefore extremely 
helpful when the desired location cannot be perceived directly from the starting position of the animal [10].

In domestic dogs, the study of spatial cognition is still at the embryonic form. One of the first studies to be conducted in domestic dogs is described by Carthy [11]. In this study, the dogs were released $6 \mathrm{~km}$ from their owner's house and their task was to walk back to their home. Over trials, the dogs gradually learned to reorient from the release point in direction to their home and to use a shorter route. Although this study suggests that dogs are able to use allocentric cues (e.g. roads, trees, etc.) to navigate within a familiar environment, it does not provide any cues about the nature of the spatial information encoded by the dogs. In a study aimed at investigating whether dogs encode egocentric or allocentric cues, Chapuis [12] used a returning task. In this experimental situation, the dog was first led along an outer path to a piece of food placed inside a bowl but the dog was refused to eat. Then, the dog was led along the same direct path to a choice point. From this position, six similar bowls (including the target one) were disposed in a semi-circle. The dog's task was to walk back to the target bowl. After several days of training, the dogs were tested in various situations: the distance between the bowl was altered, the number of bowls was doubled, the bowls were shifted to a new spatial location in the outdoor area, the dogs were carried to the choice point or the outer path was covered by a tunnel. Overall, the results suggested that dogs prefer to use allocentric cues rather than egocentric cues to return to a food location. For instance, when the bowls were translated to a new spatial position or if the number of bowls was doubled, the dogs' performance dropped significantly. However, when the egocentric frame of reference was broken, the dogs' performance was still very good. In another study [13], the dogs had to bypass two opaque or two transparent screens forming a V-shaped panel to reach a food cache (bowl). In this particular study, the length and the angle of the detour were manipulated by varying the length and position of the panel placed between the animal and the food. The results revealed that if the bowl of food was visible from the starting position, the dogs used egocentric cues and ignored the distance. However, if an opaque panel blocked the visibility of the bowl, the dogs encoded an allocentric representation and used the shortest path to reach the food. On the other hand, when their eyes are blindfolded in a search task, dogs can also rely on dead reckoning by keeping track of their displacements and reorientations in space [14]. Put together, these pioneer studies on dogs' spatial cognition suggested that dogs, like several other species, can encode and use both egocentric and allocentric cues.

More recently, Fiset and his colleagues have used an object permanence task to investigate whether dogs encode egocentric or allocentric cues in spatial working memory to navigate. More specifically, spatial working memory refers to remembering properties of locations in working memory over a short period of time. In this task (called "visible displacement problem"), which was initially developed by Jean Piaget [15] in human children, an attractive object (e.g. toy, ball) is visibly hidden behind one of several identical locations (usually three or four) and a few seconds later the animal must remember and locate the hiding position of the object. From trial to trial, however, the target object is hidden behind a different location, forcing the subject to rely on working memory to memorize different properties of locations to find the hidden object. Since several animal species face situations in which objects move and disappear (e.g. prey), the visible displacement problem is well adapted to investigate spatial working memory in animals.

The previous studies by Fiset and his colleagues showed that if there is a linear trajectory between the dog's encoding position and the position of the hidden object, the dog primarily uses egocentric cues. Most specifically, dogs encode a directional vector (including distance and direction) that starts at the animal's position and points toward the target location [6], supporting the previous work by Chapuis and her colleagues [13] in a different spatial task. However, the use of a direct path toward the hidden location is not always possible. For instance, the dog may have to bypass a brush or a pound before reaching the hiding location. By consequence, Fiset, Beaulieu, LeBlanc and Dubé [16] recently investigated spatial working memory of domestic dogs for hidden objects in a detour paradigm (for a similar approach in chicks, see $[17,18])$. Results revealed that dogs simultaneously encode both dead reckoning and allocentric spatial information when they have to bypass an obstacle to locate a hidden object. Besides when the detour involved several reorientations (U-shaped detour), over the course of time, the dogs gradually learned to rely exclusively on allocentric cues and abandoned the use of dead reckoning. This latter experiment, however, does not provide any clues about the nature of the allocentric information that is encoded in dogs' spatial working memory.

Animals mainly use two sources of allocentric spatial information to navigate: local and global cues [10,19-23]. Local cues are usually designed as objects, such as rocks, logs, or featural cues (e.g. colors) that directly marked the exact location of the desired location [21] or that are located in the vicinity of the cache [10]. As for them, global cues refer to objects that lay at a distance from the target location. In an open environment, global cues may be distant objects, such as mountains or the tree line, whereas in an enclosed space (e.g. a testing room), the geometric relationships of walls refer to the global geometry of the room and they are also frequently termed as global cues. It is usually assumed that global cues 
guide the animal toward a specific area whereas local cues are used as beacons to pinpoint a specific position within this area [24]. However, some authors assume that relationships between the global cues can also be use to pinpoint the animal towards a specific location [21].

There is evidence that animals are more sensitive to one allocentric cue than the other. For instance, in his classic studies on rats' spatial representation, Cheng [24] demonstrated that rats remembered a target location by using the global geometry of an enclosure and did not use the featural cues (local cues) added to the enclosure. Spetch and Edwards [22], however, found that in pigeons, local cues largely dominated global cues. Interestingly, when either local or global cues were made unavailable, pigeons relied on the alternative source of information to locate the food, suggesting that pigeons hierarchically encode spatial information: local cues dominate but global cues are also simultaneously encoded and used if necessary. Vallortigara, Zanforlin and Pasti [25] found a similar but inverse conclusion: Chicks could encode and use geometric (global) and featural (local) information but primarily rely on featural information when both cues are available. Readers are invited to consult Spetch and Kelly [26] who elegantly summarized how animals encode and use both local and global cues in various experimental settings.

To address the question of whether animals encode local or global cues to navigate, the landmark-based search paradigm is often used [26,27]. In this approach, an animal is trained to locate a piece of food hidden at a fixed distance and direction from one landmark or an array of landmarks within a large environment (e.g. a testing room). After training, the landmark or the array of landmarks is systematically shifted to a new location in the room, putting into conflict the vectors (distance and direction) emanating from the landmark (or from the array of landmarks) and those emanating from the walls of the room. Although pioneer works [20,28,29] suggested that birds encode distance and direction from individual vectors from landmarks only, later work by GouldBeierle and Kamil [30] suggested that birds could also encode vectors from the walls of the room. In this study, Clark's nutcrackers were trained to locate a target location between two local landmarks. On tests, although the landmarks were removed from the room and that the closest wall was located $110 \mathrm{~cm}$ from the target position, the birds still accurately located the target position.

Fiset [31,32] used the landmark-based search paradigm to investigate whether dogs encode the distance and direction from local and global cues. In these studies, a ball was hidden under a layer of woodchip located near one or two particular landmarks (a cylinder) or one or two walls. On tests, the landmark(s) was (were) shifted laterally, perpendicularly or diagonally relative to the walls of the testing chamber. The results showed that dogs encoded both the distance and the direction from the landmarks (local cues) and the walls (global cues), suggesting that they simultaneously encoded both sources of spatial information and attempted to average the vectors emanating from each cue. However, given that the landmark-based search task is a reference spatial memory paradigm (the spatial location of the hidden object is the same from trial to trial so that the information is encoded in long term memory), it still remains to determine whether dogs encode both local and global cues for a short period of time in spatial working memory.

In the current study, we used an object permanence task to investigate the nature of allocentric spatial information (local vs global cues) encoded in dogs' spatial working memory. To reach this objective, the current experiment took place in the same experimental room used by Fiset et al. [16]. In this experimental setup, the experimenter who manipulated the object and the boxes used to hide the object were termed local cues. As for them, the walls of the searching room referred to global cues. The dogs were first trained to use allocentric spatial information to find a target location in a U-shaped detour task. In tests, local and global cues were systematically put in conflict to determine which of these two sources dogs predominantly used. If dogs were primarily encoding local cues, they should have encoded the hiding position by relationships to the experimenter's position and/ or the position of the other nontarget boxes. If the dogs were encoding global cues, however, they should have searched as a function of the relationships between the walls of the searching room and the target box.

\section{MATERIAL AND METHODS}

\subsection{Animals}

The animals were seven purebred adult Labrador retrievers ( 4 females and 3 males; mean age $=4$ years and 10 month, range $=3-9$ years) that belonged to private owners. All dogs had already participated in an object permanence study, but they were all naive to the detour task used in the present experiment.

\subsection{Material}

Figure 1 illustrates the experimental room $(362 \mathrm{~cm}$ wide $\times 604 \mathrm{~cm}$ deep), which was separated in two parts by a vertical low wall ( $305 \mathrm{~cm}$ wide $\times 122 \mathrm{~cm}$ high). One part of the room $(362 \mathrm{~cm}$ wide $\times 250 \mathrm{~cm}$ deep) served as the searching room where the target object was hidden by E1 (Experimenter 1). The dog and E2 (Experimenter 2) were located in the second part of the room $(362 \mathrm{~cm}$ wide $\times 354 \mathrm{~cm}$ deep). The dog sat in front of an opening $(85 \mathrm{~cm}$ high $\times 57 \mathrm{~cm}$ wide) made in the vertical wall, and E2 knelt at the left side of the dog. The center of this 


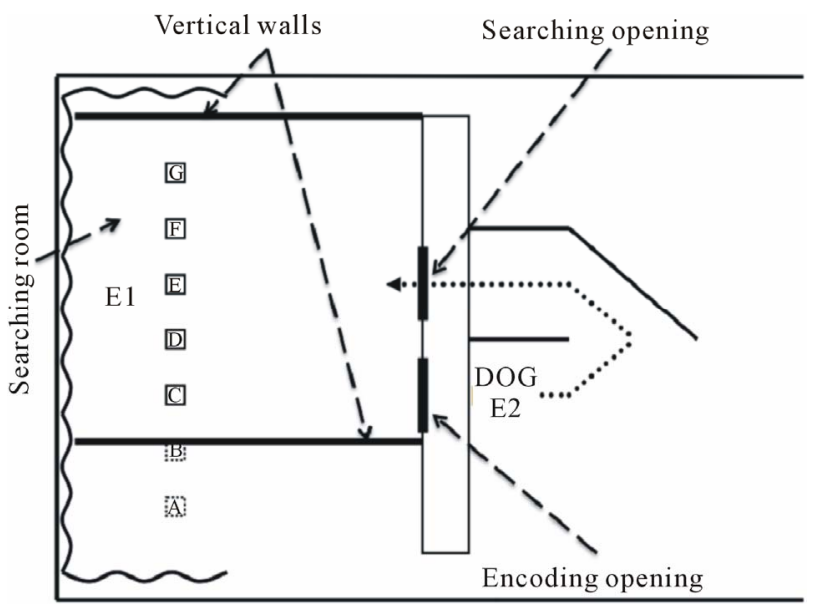

Figure 1. Schematic representation of the testing room. E1 = experimenter who performed the manipulations, E2 = experimenter who held the dog. A, B, C, D, E, F, and G represent the seven potential positions of the three adjacent hiding boxes. Dotted line: U-shaped pathway used by the dog. Drawing not at scale.

opening (called "encoding opening") was located $127 \mathrm{~cm}$ from the left end of the vertical wall. An opaque sliding door $(90 \mathrm{~cm}$ high $\times 59 \mathrm{~cm}$ wide) slid from the left into the encoding opening. A second opening (called "searching opening", $85 \mathrm{~cm}$ high $\times 57 \mathrm{~cm}$ wide) was made in the vertical wall and from center to center, the linear distance between the encoding and the searching openings was $65 \mathrm{~cm}$. To access the searching room through the searching opening, the dog had to follow a U-shaped pathway created by a series of low walls placed on the floor of the encoding part of the room. A first wall (122 $\mathrm{cm}$ high $\times 142 \mathrm{~cm}$ wide) was placed perpendicularly between the encoding and the searching opening. A second wall (122 cm high $\times 142 \mathrm{~cm}$ wide) was placed perpendicularly $10 \mathrm{~cm}$ at the right side of the searching opening. Finally, a third wall $(122 \mathrm{~cm}$ high $\times 142 \mathrm{~cm}$ wide) was placed diagonally at the far end of the second low wall, creating, from the encoding position of the dog, a U-shaped pathway leading to the search area. To prevent the dog from seeing the position of the moving walls in the searching area, a flat panel $(305 \mathrm{~cm}$ wide $\times$ $33 \mathrm{~cm}$ deep) covered the top of the vertical low wall.

Three identical rectangular opaque boxes $(12 \mathrm{~cm}$ wide $\times 30 \mathrm{~cm}$ high $\times 12 \mathrm{~cm}$ deep with a top, a front, and two side panels) served as potential hiding boxes. The three boxes always occupied adjacent positions and were fixed on a transparent plexiglass sheet $(92 \mathrm{~cm}$ long $\times 12 \mathrm{~cm}$ wide) at a distance of $28 \mathrm{~cm}$ from each other (from edge to edge). The boxes could occupy seven positions (A, B, C, D, E, F, G) in the searching room: One position (D) was in front of the center of the vertical wall, three (A, B, C) were to the left of this central position, and three (E, F, $\mathrm{G})$ were to the right. These positions were arrayed in a row $28 \mathrm{~cm}$ apart, and $160 \mathrm{~cm}$ from the vertical wall. Position $\mathrm{C}$ was positioned in front of the encoding opening and Position E was located in front of the searching opening.

A white Masonite sheet $(244 \mathrm{~cm}$ wide $\times 209 \mathrm{~cm}$ high $)$ was placed on each side of the searching room, providing lateral walls. These sheets were suspended $1 \mathrm{~cm}$ above the floor and were $228 \mathrm{~cm}$ apart. Four small rubber wheels were placed on a track fixed to the ceiling of the room and served to move the lateral walls as silently as possible $80 \mathrm{~cm}$ to the right or to the left. The mobile walls were connected by three transversal sticks $(8 \mathrm{~cm}$ wide $\times 244 \mathrm{~cm}$ long) and could therefore be moved simultaneously. An opaque white curtain hanging from the ceiling of the experimental room provided a uniform visual background behind E1 and the walls. A fluorescent light suspended above the dog's starting position lit the room. Since a large panel covered the top of the vertical low wall, this light was imperceptible to the dog from the opening or the searching opening.

One experimenter (E1) performed the manipulation and positioned herself behind the central box in the searching area. The second experimenter (E2) remained outside of the searching area near the central opening where she restrained the dog by holding its collar during the manipulation of the target object.

\subsection{Procedure}

The experiment was divided into three successive steps: shaping, training and testing. To prevent dogs from using olfaction for finding the hidden object, rose water (diluted 1/10) was sprayed over the boxes every four trials. The use of rose water is known as a very effective mean for masking olfaction in dogs [6,16,33-36]. In addition, the dogs were forced to make a U shaped detour in order to control for the use of linear egocentric cues that could be used to locate the hidden object. All dogs were unfamiliar with the two experimenters.

\subsection{Shaping}

Although all dogs demonstrated a strong motivation by the opportunity to grab the target object, a food reinforcement procedure was introduced to train the dogs to touch the target object.

At the beginning of a shaping trial, the dog sat in front of the encoding opening and E2 sat on the left side of the animal. E1 put down the target object in front of the central box while E2 restrained the animal by grasping its collar. Then, E1 lifted up the object with her hand, captured the dog's attention, moved the object visibly in front of each of the three boxes, and finally placed the object between two boxes (but never behind). If the dog did not watch the movement throughout all the sequence, 
the trial was interrupted and repeated. Because the dogs were highly motivated to play with the target object, repeated trials rarely occurred. Once the object was put down, E1, to prevent cuing, looked straight ahead over the low vertical wall. As soon as the object was put down by E1, E2 slid the door in front of the encoding opening for a retention interval of $10 \mathrm{~s}$. The purpose of this manoeuvre was to habituate the dogs to the delay that was used later in Testing. At the end of the interval, E2 released the dog, which had to follow the U-shaped pathway to enter the searching room. At the beginning of shaping, E2 had to gently guide the dog through the U-shaped pathway but after a few trials, this procedure was no longer necessary. The dog was reinforced by E1 if one of the following behaviors was exhibited in the minute that followed its release: Grasping the object with its mouth, touching it with its paw or putting its muzzle on it. A piece of commercial dry food (Diet NutriScience) and social rewards (strokes, verbal rewards such as "Good dog!") were used as reinforcements.

In shaping, we made sure that the dog touched the target object between each of the seven positions where the boxes could be placed in the searching room. Within a shaping session, the position of the entire experimental layout (the mobile walls, the boxes and E1) was randomly varied from trial to trial. During the 30 -s intertrial interval, to make sure that the dog did not perceive the displacement of the experimental setting from one position to the other, E1 slid the door in front of the encoding opening. Each shaping session included 30 trials and we ended the shaping phase when the dog had touched the target object on 10 consecutives trials.

\subsection{Training}

The dogs were trained to use allocentric spatial information (E1, boxes and walls) to locate the target object in the U-shaped detour task. To do so, after the object disappearance inside one of the three boxes, out of the dog's view, the walls, the boxes, the target object and E1 were all moved $80 \mathrm{~cm}$ to the right or to the left on a new spatial position. Consequently, within a trial, because the distance and the angular deviation between each of these objects were kept constant, the spatial relationships between E1, the three boxes and the two mobile walls always stayed the same. After the displacement of those allocentric cues, the dog was allowed to make its search by using the U-shaped detour. By moving the allocentric cues between encoding and searching, we assured that the dogs would not encode a sophisticated source of egocentric information (dead reckoning) in spatial working memory.

Figure 2 illustrates an example of a particular training trial. Positions A-B were unoccupied, the boxes were placed in Positions C-D-E and two other unoccupied positions (Position $\mathrm{F}$ and $\mathrm{G}$ ) were hidden by the mobile walls. E1 was situated behind the box located in Position C. At the beginning of the trial, E1 showed the object to the dog and hid it inside the target box located in Position C. Then, E2 closed the sliding door in front of the encoding opening for a 10 -s retention interval. Next, out of the dog's view, E1 moved the three boxes, the object, and the mobile walls to two adjacent positions to the right, and she replaced herself behind the box located in Position E. Then, E2 released the dog, which turned through 180 degrees to follow the U-shaped pathway leading to the search area. The dog was reinforced if it found the target object inside the first box visited. To locate the hidden object, the dog had to rely on one or several sources of allocentric information available in the search area, that is, the relationships between the target box and the boxes, the experimenter and/or the mobile walls. As described in the shaping phase, E1 reinforced with food and social rewards if it grasped or touched the target object.

Within each training session, the target object was randomly hidden six times at each of the three central positions (D, E, F) for a total of 18 trials and the target position was never the same on more than two consecutive trials. Within each session, at the beginning of each trial, the mobile walls were positioned nine times on the right side of the searching room and nine times on the left side, and the three boxes were always placed on Po-

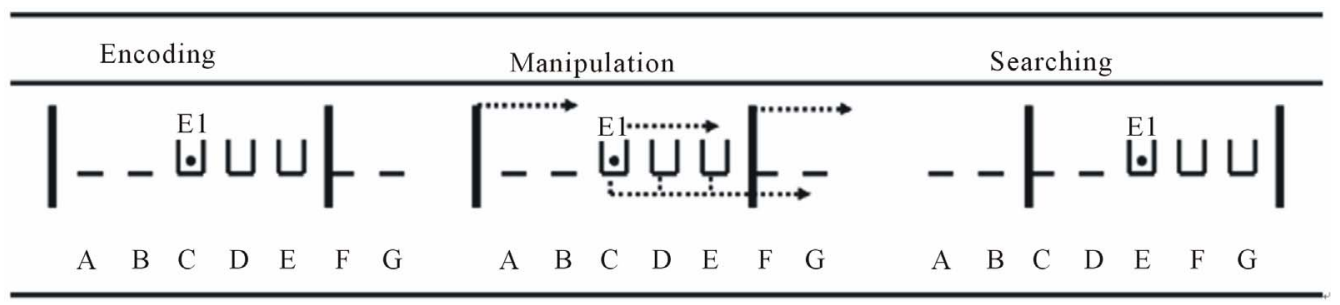

Figure 2. Example of a training trial. All cues were moved to a new position but the relationships between the walls (vertical bars), the boxes (open boxes), E1, and the target object (black dot) did not change before (encoding), during (manipulation), and after (searching) a trial. The dotted lines exhibit the displacement and the arrows show the direction and destination. A, B, C, D, E, F, and G represent the seven potential positions of the three adjacent hiding boxes; $\mathrm{E} 1=$ the experimenter who performed the manipulations. 
sition D-E-F. From trial to trial, to make sure that the dogs did not perceive the displacement of the experimental setting from one position to the other, the encod ing searching door was closed during the 30 -s intertrial interval.

To complete the training phase, the dogs had to succeed on at least 15 trials out of 18 during two consecutives sessions. Moreover, we removed the dog from the study if for 5 consecutive training sessions the dog did not succeed at least 11 trials out of 18 in one session. Based on a binomial test $(p=0.33)$, this level of performance was over chance.

\subsection{Testing}

Testing began the day after the end of training. Each test session began with three trials similar to those used in the shaping phase. Consequently, the target object was simply placed between two boxes in full view of the dog and it was allowed to touch it by using the U-shaped tunnel. These three trials were then followed by test trials. On each testing trial, E1 hid the target object inside one of the three boxes, as was done during training. Then, E2 slid the opaque door in front of the encoding opening for a 10-s interval. During this short retention interval, E1 performed an experimental or control trial. At the end of the retention interval, E2 released the dog, which followed the U-shaped pathway leading to the boxes. As described in the shaping phase, if the dog grasped or touched the object, E1 reinforced it with food and social rewards.

We introduced two types of experimental trials: local and global trials. On local trials, the three boxes, E1 and the target object were all moved $80 \mathrm{~cm}$ to the right or to the left between encoding and searching. The mobile walls remained at their initial position. In the example of the local trial illustrated in Figure 3, Positions A-B were unoccupied at the beginning, the three boxes were in Positions C-D-E; the right wall hid Positions F-G and E1 was standing behind Position E. E1 showed the object to the dog and hid the target object inside the box in Position E. Then, E2 closed the sliding opaque door in front of the encoding opening for 10-s. During this retention interval, the array of boxes, the target object and E1 were moved two positions to the right $(80 \mathrm{~cm})$. To control for the noise made in global trials (see below), E1 lifted up the target object and put it back inside the target box. She also moved the mobile walls and returned them back in place. At the end of the 10-s interval, E2 released the dog, which took the U-shaped pathway. When the dog entered the searching room, Positions A-B were still hidden by the left wall but Positions C-D were now unoccupied and the boxes were in Positions E-F-G, and E1 and the target object were in Position G. If the dogs located the position of the hiding box by using local cues (the relationships

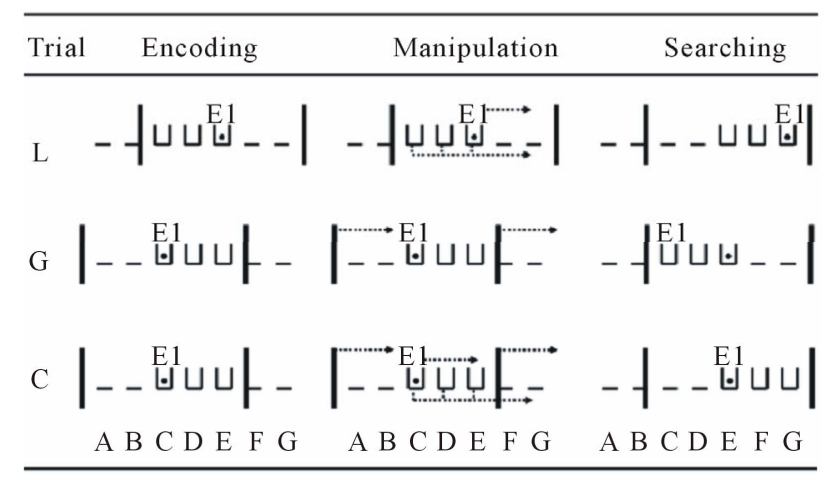

Figure 3. Example of a local (L) trial, a global (G) trial, and a control (C) trial in the testing phase. Depicted are the positions of the walls (vertical bars), the boxes (open boxes), E1, and the target object (black dot) before (encoding), during (manipulation), and after (searching) a trial. The dotted lines exhibit the displacement and the arrows show the direction and destination. $\mathrm{A}, \mathrm{B}, \mathrm{C}, \mathrm{D}, \mathrm{E}, \mathrm{F}$, and $\mathrm{G}=$ potential positions of the boxes; $\mathrm{E} 1=$ the experimenter who performed the manipulations.

between the target box and/or the position of E1), they should have searched for the object at its true location, that is, inside the box in Position G, and succeeded. However, if they used global cues, the dogs should have searched inside the box located in Position E, and failed.

On global trials, the two mobile walls and the target object, but not the boxes and E1, were moved $80 \mathrm{~cm}$ to the right or to the left between encoding and searching. To control for the noise made in the local trials, E1 moved and returned to her position and she also moved the array of boxes and put it back in place before the end of the 10-s retention interval. If dogs located the target position by using the walls of the room in the global trial illustrated in Figure 3, they should have searched for the target object in Position E, and succeeded. However, if they encoded local cues, the dogs should have searched for the object in Position C, and failed.

On control trials, the target object could be found by using either local or global cues. In the control trials, the two mobile walls, the boxes, E1 and the target object were all in the same position before and after the 10-s retention interval. However, to control for the noise made in experimental trials, E1 moved the mobile walls and the boxes to the right or to the left and put them back in place before the end of the retention interval. E1 also moved and returned to her initial position. Finally, E1 lifted up the target object and put it back into the hiding box. In the control trial illustrated in Figure 3, if they used local and/or global cues, the dogs should have searched behind the box located in Position E and succeeded.

Each type of trial (control, local and global) was administered in a separate session of 30 trials to each dog: thus, each dog performed 30 control, 30 local and 30 global trials. The order in which the dogs received the 
testing sessions was counterbalanced among the dogs. Within a session, the two positions of the mobile walls (A-E and C-G) were used an equal number of times at the beginning of the trials. Moreover, the object was initially hidden the same number of times in Positions $\mathrm{C}$ and $\mathrm{E}$ in a random order and each trial was separated by a 30-s intertrial interval.

\section{RESULTS}

In shaping, the seven dogs took a mean number of 1.72 sessions $(S E=0.27)$ to reach the criterion of 10 consecutive successful trials. In training, although there was no trial without a search attempt, suggesting that all dogs were highly motivated to search for the hidden object, four dogs out of seven did not reach the training criterion and thus did not complete the training phase. These four dogs ( 1 male and 3 females) were trained for a total of 5 to 8 sessions and their mean percentage of success was $50.00 \%(S E=1.60)$ in their last two training sessions. Moreover, two of these four dogs performed over chance in only one training session and the two remaining dogs never performed over chance in a single session. Thus, these dogs did not proceed to testing. A closer look at the search behavior of these four dogs did not reveal any evidence of using an egocentric strategy (e.g. dead reckoning) to solve the detour task during the training phase.

As for the three successful dogs, they required an average of 11.33 sessions ( $S E=3.84$, range 4 to 17 sessions) to reach the training criterion. Given the small number of $\operatorname{dogs}(N=3)$ that continued to the testing phase, it was extremely hazardous to statistically compare the performance of dogs among the three types of testing trial. Consequently, we analyzed the dogs' performance individually for each type of trial. We used a binomial test ( $p$ $=0.33, n=30$ trials) to determine whether dogs' performance in each type of trial differed significantly from the number of successful trials expected by chance; 16 successful trials or higher was significantly different from chance. On the basis of this criterion, all dogs succeeded on control trials but each dog failed the global and local tests (see Figure 4). A series of one-sample $t$ tests on the mean number of successful trials also confirmed that the three dogs, as a group, performed over chance $(p=.33)$ in control trials $[\mathrm{M}=81.1 \%, \mathrm{SD}=15.8]$ $(t(2)=5.25, p=0.034)$ but at chance level in local $[\mathrm{M}=$ $28.8 \%, \mathrm{SD}=8.4](t(2)=-0.59, p=0.618)$ and global trials $[\mathrm{M}=32.2 \%, \mathrm{SD}=16.4](t(2)=-0.23, p=0.84)$.

To determine whether the dogs' performance varied during the course of testing, for each dog, we divided each testing session in 3 blocks of 10 trials (see Figure 5). As one can see, the dogs' performance varied substantially between the first and second block. However, it remained relatively stable during the last two blocks for

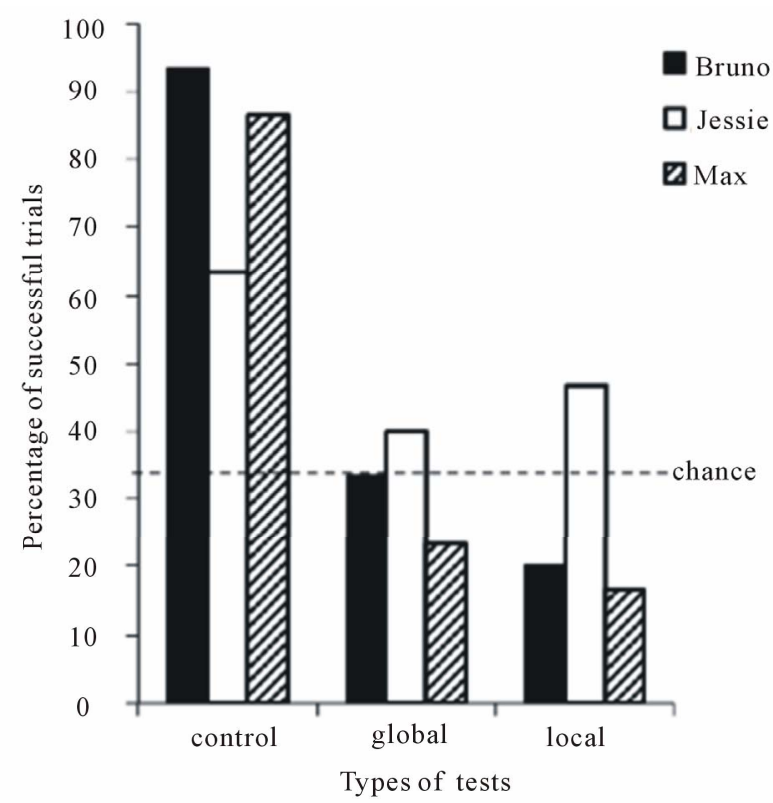

Figure 4. Percentage of successful trials as a function of each type of test for each dog.

each type of trial. Moreover, in the third block of trials, each dog performed over chance in the control trials (Bruno, $p=0.006$; Jessie, $p=0.006$; Max, $p=0.006$ ) but each dog failed the local (Bruno, $p=0.61$; Jessie, $p=$ 0.15 ; Max, $p=0.036$ [below chance]) and global trials (Bruno, $p=0.86$; Jessie, $p=0.41$; Max, $p=1.00$ ). Put together, these results on dogs' success support the conclusion that the dogs did not use any particular type of allocentric cues to find the hidden object in the detour task.

To further determine whether the dogs used a specific search strategy during the experimental trials, we also examined the distribution of dogs' search attempts in the searching room. First, it should be noted that the encoding and searching openings were the only features that remained at a stable position in the room from trial to trial. Based on this information, one could argue that the dogs could have used these stable cues to search for the hidden object. More specifically, when exiting from the detour, instead of searching as a function of one source of allocentric information, the dogs could have chosen the closest box to the searching opening. To test this alternative hypothesis, we examined the spatial distribution of search attempts made by each dog at each potential hiding location in the searching room as a function of the position of the searching opening. In addition, to get a global picture of the spatial distribution of all search attempts, both correct and incorrect responses were pooled in these analyses.

In the local trials, after the displacement of E1 and the boxes to a new position, the cues (boxes, E1 and walls) could occupy two spatial configurations. In the first con- 

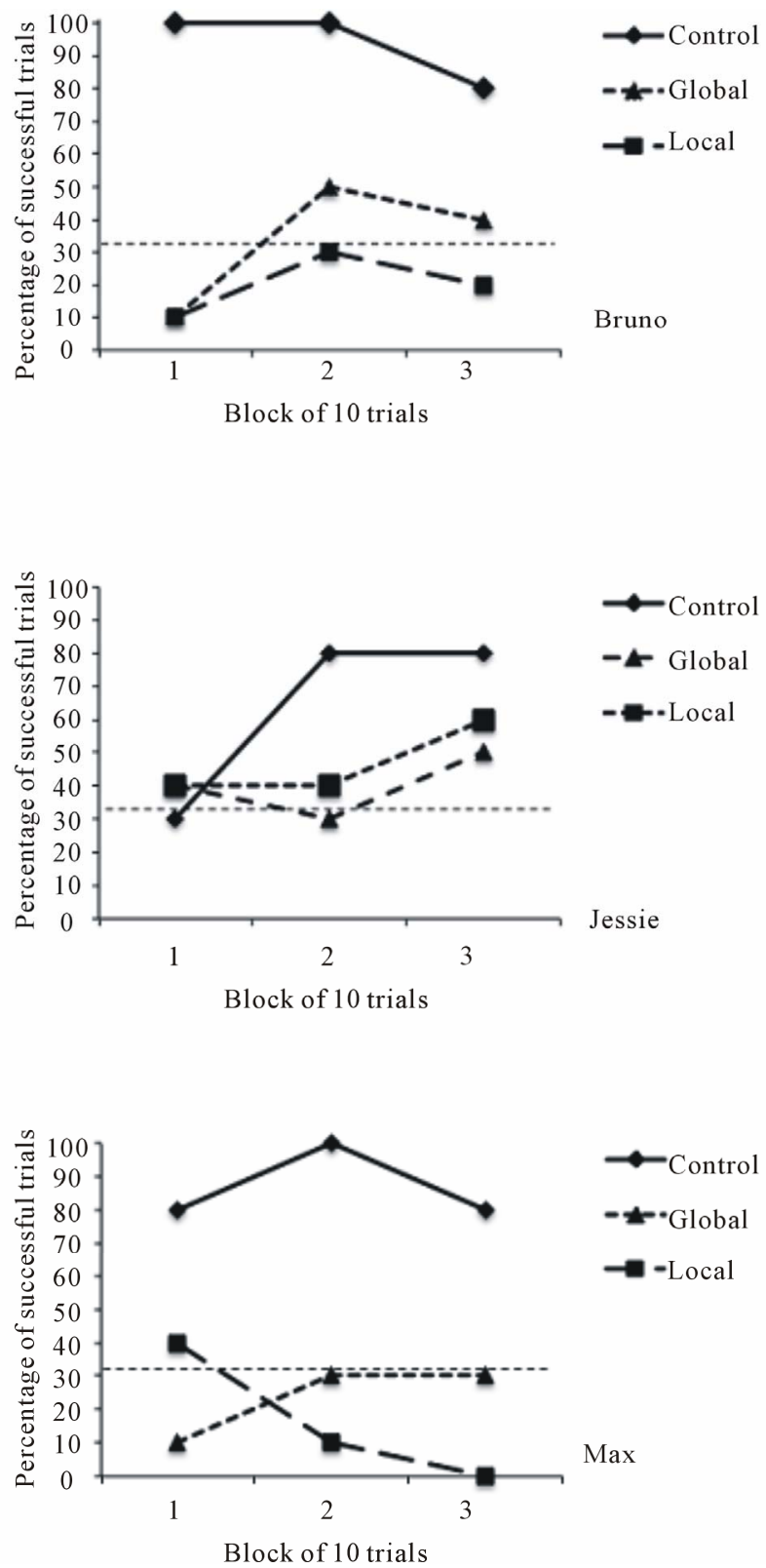

Figure 5. Percentage of successful trials as a function of each type of test and blocks of 10 trials for each dog. Dotted line $=$ chance $(33.3 \%)$.

figuration, the boxes were on position $\mathrm{A}, \mathrm{B}, \mathrm{C}$, positions $\mathrm{D}$ and $\mathrm{E}$ were empty and a moving wall hid positions $\mathrm{F}$ and $\mathrm{G}$. In the second configuration, the boxes were on position E, F, G, positions D and E were empty and a moving wall hid positions A and B. As one can see in Table 1, in the second configuration of the local trials, two dogs significantly searched at the closest box to the searching opening. However, since they did not show this pattern of search distribution in the first configuration (actually, one dog-Jessie - significantly searched more frequently at the farthest position to the searching opening), it rather seems that the dogs did not use this
Table 1. Number of search attempts made by each dog to each box in the searching room as a function of both spatial configurations in the local trials.

\begin{tabular}{ccccccccccc}
\hline \multicolumn{1}{c}{ Configuration 1 } & \multicolumn{4}{c}{ Configuration 2 } \\
\hline & C & D & E & F & G & A & B & C & D & E \\
Jessie & - & - & 0 & 1 & $14^{*}$ & 0 & 2 & $13^{*}$ & - & - \\
Bruno & - & - & 8 & 7 & 0 & 6 & 6 & 3 & - & - \\
Max & - & - & 3 & 7 & 5 & 0 & 1 & $14^{*}$ & - & - \\
\hline${ }^{*} p<0.05$, Binomial test $(n=15, p=0.33)$. & & & &
\end{tabular}

particular strategy in the local trials.

In the global trials, there were also two possible spatial configurations after the displacement of the walls in the searching room. In the first configuration, the boxes were on position $\mathrm{C}, \mathrm{D}, \mathrm{E}$, positions $\mathrm{A}$ and $\mathrm{B}$ were empty and a moving wall hid positions $\mathrm{F}$ and $\mathrm{G}$. In the second configuration, the boxes were also on position $\mathrm{C}, \mathrm{D}, \mathrm{E}$, but positions $\mathrm{F}$ and $\mathrm{G}$ were empty and a moving wall hid positions A and B. Table 2 shows that one dog (Jessie) searched significantly at the box closest to the searching opening in both configurations. However, the two other dogs significantly searched to different locations in the first configuration and their search attempts were almost equally distributed to the three boxes in the second configuration.

Put together, these analyses of search distribution in the local and global trials suggest that one dog (Jessie) seemed to search as a function of the closest box to the searching opening. However, a closer look at the distribution of its search attempts in the searching room revealed a more parsimonious explanation: this dog searched for the hidden object to the box adjacent to the right wall of the room. Indeed, this dog made a total of 49 search attempts to the box adjacent to the right wall out of 60 experimental trials. The two other dogs, however, did not show any evidence of using a particular search strategy and they seemed to search randomly behind one of the three boxes available in the searching room.

\section{DISCUSSION}

The aim of this study was to determine whether domestic dogs encode local and/or global cues in spatial working memory. To reach this object, seven Labrador retrievers were trained to use a source of local allocentric cues (boxes and/or experimenter) or of global allocentric cues (walls of the room) to find an attractive object they saw disappear inside an opaque box. Interestingly, out of the seven dogs that were trained, solely three learned to use the allocentric cues to find the hidden object. In the tests, when the local and global cues were put in conflict, the three dogs were unable to rely exclusively on one particular source of allocentric information to successfully locate the hidden object: two dogs searched ran- 
Table 2. Number of search attempts made by each dog to each box in the searching room as a function of both spatial configurations in the global trials.

\begin{tabular}{ccccccccccc}
\hline \multicolumn{1}{c}{ Configuration 1 } & \multicolumn{4}{c}{ Configuration 2 } \\
\hline & C & D & E & F & G & A & B & C & D & E \\
Jessie & 2 & 1 & $12^{*}$ & - & - & - & - & 0 & 5 & $10^{*}$ \\
Bruno & 3 & $10^{*}$ & 2 & - & - & - & - & 8 & 7 & 0 \\
Max & $12^{*}$ & 1 & 2 & - & - & - & - & 6 & 4 & 5 \\
\hline${ }^{*} p<0.05$, Binomial test $(n=15, p=0.33)$. & & & &
\end{tabular}

" $p<0.05$, Binomial test $(n=15, p=0.33)$.

domly among the three boxes placed in the room and one dog systematically searched to the box adjacent to one wall.

Why did dogs fail to locate the disappearing object in the local and global tests? One possible explanation is that the dogs did not take into account the dissociation between local and global cues and perceived those cues as a single frame of reference. During training, the dogs might have learned to locate the target position by using a configuration of all allocentric cues available in the room (e.g. the boxes, the experimenter and the walls). Consequently, when the allocentric cues were put in conflict, the configuration was broken and the dogs failed to locate the hiding position: they behaved as if they were entering a new testing chamber after taking the U-shaped detour. This interpretation of dogs' spatial working memory is in discordance with the one proposed by Fiset $[31,32]$ who suggested that dogs encode and average in spatial reference memory individual vectors emanating from landmarks and walls in a testing room. In the current study, if the dogs had encoded in working memory individual vectors from the allocentric cues when one of those cues were shifted to a new location, the dogs should have searched at an intermediate position between the position pointed by the cues that were shifted and those that were not. Our dogs, however, did not search as a function of the displacement of the local or global cues: either they searched along a particular wall or they searched randomly. It therefore seems that dogs encode in spatial working memory a configuration of cues whereas they encode individual vectors in spatial reference memory. To shed some light on this discrepancy between spatial working and reference memory in dogs, further studies are therefore called for investigating dogs' spatial working and reference memory in a similar experimental paradigm. Besides, it should be noted that the distinction between local and global cues was arbitrary determined by us at the beginning of the current study and it may be possible that dogs do not perceive this dissociation as humans do. However, given that other mammal species have demonstrated the ability to encode either local or global cues $[10,23,33]$, it would be quite surprising if dogs could not discriminate these two sources of allocentric cues.
Another plausible explanation for the current results refers to the relationship between the size of our testing room and the size of our subjects. Indeed, our dogs (Labrador retrievers) were large animals (roughly $100 \mathrm{~cm}$ length) tested in a relatively small room $(228 \mathrm{~cm}$ wide $\times$ $250 \mathrm{~cm}$ deep). Consequently, the global cues (walls of the room) were possibly too close from the encoding position of the animal to be considered as distant or global cues. The dogs could have perceived all the allocentric cues that were available in the room (walls, boxes, experimenter) as local cues. Recent evidence supports this hypothesis. For instance, Sovrano et al. [37] showed that fish do not encode the same spatial cues when tested in a small or a large-scale enclosure. Therefore, it would be interesting to test domestic dogs in a larger environment to determine whether the size of a room influences the encoding of local and global cues when they search for a disappearing object in a detour task. Nevertheless, it should be noted that Fiset and Doré [7] demonstrated that domestic cats, despite the fact that they were tested in a room smaller than the one we used in the present study, dissociate the boxes (local cues) from the walls (global cues) when searching for a hidden object in a detour task.

In the current study, the spatial working memory task was extremely difficult to master. Indeed, in training trials, between encoding and searching, the walls, the boxes and the experimenter were all moved to a new position, forcing the dogs to rely exclusively on allocentric information to locate the hidden object. This resulted in a situation where four out of our seven dogs failed to reach the testing phase. It therefore seems that the encoding of allocentric cues in dogs' spatial working memory to localize of a disappearing object in a detour situation is a very demanding process. Consequently, when those allocentric cues were put in conflict in the experimental tests, the dogs were extremely confused by the manipulation and failed to use a specific cue to find the object. This latter observation supports previous findings [16] that showed that domestic dogs demonstrate a higher level of success for finding a disappearing object in a detour task when they can encode and use simultaneously multiple sources of spatial information (e.g. dead reckoning and allocentric information). When they have to rely on one particular source (allocentric cues), as in the current study, the localization of a hidden object in spatial working memory is particularly tough and the capacity to find the object dropped significantly.

Future studies should also investigate the possibility that dogs encode a snapshot of the allocentric cues to locate a hiding position. This hypothesis predicts that dogs would learn to associate the allocentric cues projected on their visual receptors and would store this representation as a snapshot. In search for the hidden object, dogs would attempt to match the perceptual image of the cues and its 
encoded snapshot. A similar process has already been proposed by [38] to explain how bees locate a source of food placed at a fixed location from a configuration of landmarks. However, other studies suggest that mammals use different mechanisms such as the absolute [38,39] or relative distances $[40,41]$ between the different cues to pinpoint a target location and additional studies are needed to dissociate these mechanisms.

Finally, the current study should be ideally repeated with more animals to increase the sample size. However, due to some major renovations that took placed into the laboratory room since the end of the study, it is materially impossible to replicate the experimental setup used in the current study in our laboratory. But one thing is sure: the present study revealed that domestic dogs have difficulties to encode allocentric cues in spatial working memory. When searching for a disappearing object in a small-scale environment, dogs do not dissociate local and global allocentric cues. When those cues are put into conflict, the domestic dogs are uncertain as to what cue use. Further studies are therefore called for investigating how dogs encode local and global cues in a large-scale environment.

\section{ACKNOWLEDGEMENTS}

This research was supported by an operating research grant from the Natural Sciences and Engineering Research Council of Canada (NSERC) and by a research grant from the Faculté des Études Supérieures et de la Recherche (FESR) of Université de Moncton. The experiment received approval from the Comité de protection des animaux from the Faculté des études supérieures et de la recherche de l'Université de Moncton, which is responsible for the application and enforcement of rules of the Canadian Council on Animal Care. An Undergraduate Student Research Award from NSERC supported Nathalie Malenfant. We thank the owners of the dogs who participated in these experiments. Finally, a special thank you to Vickie Plourde who made constructive comments on a previous version of this manuscript and to one reviewer who suggested analysing the distribution of search attempts as a function of the position of the searching opening.

\section{REFERENCES}

[1] Hauser, M.D. (2000) Wild minds: What animals really think. Henry Holt, New York.

[2] Pearce, J. (1997) Animal learning and cognition: An introduction. 2nd Edition, Psychology Press, Hove.

[3] Roberts, W.A. (1998) Principles of animal cognition. McGraw-Hill, Boston.

[4] Shettleworth, S.J. (2010) Cognition, evolution, and behavior. 2nd Edition, Oxford University Press, Oxford.

[5] Thinus-Blanc, C. (1996) Animal spatial cognition: Behavioural and brain approach. World Scientific, Singapore.

[6] Fiset, S., Landry, F. and Ouellette, M. (2006) Egocentric search for disappearing objects in domestic dogs: Evi- dence for a geometric hypothesis of direction. Animal Cognition, 9, 1-12. doi:10.1007/s10071-005-0255-1

[7] Fiset, S. and Doré, F.Y. (1996) Spatial encoding in domestic cats (Felis catus). Journal of Experimental Psychology: Animal Behavior Processes, 22, 420-437. doi:10.1037/0097-7403.22.4.420

[8] Nadel, L. (1990) Varieties of spatial cognition: Psychobiological considerations. In Diamond, A., Ed., The Development and Neural Bases of Higher Cognitive Functions, Academic Press, New York, 613-626.

[9] Pick, H.L. and Lockman, J.J. (1981) From frames of reference to spatial representations. In: Liben, L.S., Patterson, A.H. and Newcombe, N., Eds., Spatial Representation and Behavior across the Life Span: Theory and Application, Academic Press, New York, 39-61.

[10] Vlasak, A.N. (2006) Global and local spatial landmarks: Their role during foraging by Columbian ground squirrels (Spermophilus columbianus). Animal Cognition, 9, 71-80. doi:10.1007/s10071-005-0006-3

[11] Carthy, J.D. (1956) Animal navigation; how animals find their way about. G. Allen \& Unwin, London.

[12] Chapuis, N. (1982) Referentiels spatiaux utilises dans la réalisation d'un trajet inverse chez le chien. L'année Psychologique, 82, 75-100. doi:10.3406/psy.1982.28409

[13] Chapuis, N., Thinus-Blanc, C. and Poucet, B. (1983) Dissociation of mechanisms involved in dogs' oriented displacements. Quarterly Journal of Experimental Psychololy $B$, 35, 213-219.

[14] Cattet, J. and Etienne, A.S. (2004) Blindfolded dogs relocate a target through path integration. Animal Behaviour, 68, 203-212. doi:10.1016/j.anbehav.2003.11.007

[15] Piaget, J. (1954) The construction of reality in the child. Basic Books, New York.

[16] Fiset, S., Beaulieu, C., LeBlanc, V. and Dubé, L. (2007) Spatial memory of domestic dogs (Canis familiaris) for hidden objects in a detour task. Journal of Experimental Psychology: Animal Behavior Processes, 33, 497-508. doi:10.1037/0097-7403.33.4.497

[17] Regolin, L., Vallortigara, G. and Zanforlin, M. (1995) Detour behaviour in the domestic chick: Searching for a disappearing prey or a disappearing social partner. Animal Behaviour, 50, 203-211. doi:10.1006/anbe.1995.0232

[18] Regolin, L., Vallortigara, G. and Zanforlin, M. (1995) Object and spatial representations in detour problems by chicks. Animal Behaviour, 49, 195-199. doi:10.1016/0003-3472(95)80167-7

[19] Church, D.L. and Plowright, C.M.S. (2006) Spatial encoding by bumblebees (Bombus impatiens) of a reward within an artificial flower array. Animal Cognition, 9, 131-140. doi:10.1007/s10071-005-0011-6

[20] Gould-Beierle, K.L., \& Kamil, A.C. (1996) The use of local and global cues by Clark's nutcrackers, Nucifraga columbiana. Animal Behaviour, 52, 519-528. doi:10.1006/anbe.1996.0194

[21] Sherry, D.F. (1992) Landmarks, the hippocampus, and spatial search in food-storing birds. In: Honig, W.K. and Fetterman, J.G., Eds., Cognitive Aspects of Stimulus Control, Erlbaum Associates Publishers, Hillsdale, 185-201. 
[22] Spetch, M.L. and Edwards, C.A. (1988) Pigeons, Columba-livia, use of global and local cues for spatial memory. Animal Behaviour, 36, 293-296. doi:10.1016/S0003-3472(88)80274-4

[23] Vlasak, A.N. (2006) The relative importance of global and local landmarks in navigation by Columbian ground squirrels (Spermophilus columbianus). Journal of Comparative Psychology, 120, 131-138. doi:10.1037/0735-7036.120.2.131

[24] Cheng, K. (1986) A purely geometric module in the rat's spatial representation. Cognition, 23, 149-178. doi:10.1016/0010-0277(86)90041-7

[25] Vallortigara, G., Zanforlin, M. and Pasti, G. (1990) Geometric modules in animals \& apos; spatial representations: A test with chicks (Gallus gallus domesticus). Journal of Comparative Psychology, 104, 248. doi:10.1037/0735-7036.104.3.248

[26] Spetch, M.L. and Kelly, D.M. (2006) Comparative spatial cognition: Processes in landmark- and surface-based place findings. In: Wasserman, E.A. and Zentall, T.R., Eds., Comparative Cognition: Experimental Explorations of Animal Intelligence, Oxford University Press, Toronto, 210228.

[27] Cheng, K. and Spetch, M.L. (1998) Mechanisms of landmark use in mammals and birds. In: Healy, S.A., Ed., Spatial Representation in Animals, Oxford University Press, Oxford, 1-17.

[28] Cheng, K. (1989) The vector sum model of pigeon landmark use. Journal of Experimental Psychology: Animal Behavior Processes, 15, 366-375. doi:10.1037/0097-7403.15.4.366

[29] Cheng, K. and Sherry, D.F. (1992) Landmark-based spatial memory in birds (parus-atricapillus and columba-livia) - The use of edges and distances to represent spatial positions. Journal of Comparative Psychology, 106, 331341. doi:10.1037/0735-7036.106.4.331

[30] Gould-Beierle, K.L. and Kamil, A.C. (1999) The effect of proximity on landmark use in Clark's nutcrackers. Animal Behaviour, 58, 477-488. doi:10.1006/anbe.1999.1185

[31] Fiset, S. (2007) Landmark-based search memory in the domestic dog (Canis familiaris). Journal of Comparative Psychology, 121, 345-353. doi:10.1037/0735-7036.121.4.345

[32] Fiset, S. (2009) Evidence for averaging of distance from landmarks in the domestic dog. Behavioural Processes, 81, 429-438. doi:10.1016/j.beproc.2009.03.016

[33] Fiset, S., Gagnon, S. and Beaulieu, C. (2000) Spatial encoding of hidden objects in dogs (Canis familiaris). Journal of Comparative Psychology, 114, 315-324. doi:10.1037/0735-7036.114.4.315

[34] Gagnon, S. and Doré, F.Y. (1992) Search behavior in various breeds of adult dogs (Canis familiaris): Object permanence and olfactory cues. Journal of Comparative Psychology, 106, 58-68. doi:10.1037/0735-7036.106.1.58

[35] Gagnon, S. and Doré, F.Y. (1993) Search behavior of dogs (Canis familiaris) in invisible displacement problems. Animal Learning \& Behavior, 21, 246-254. doi:10.3758/BF03197989

[36] Gagnon, S. and Doré, F.Y. (1994) Cross-sectional study of object permanence in domestic puppies (Canis familiaris). Journal of Comparative Psychology, 108, 220-232. doi:10.1037/0735-7036.108.3.220

[37] Sovrano, V.A., Bisazza, A. and Vallortigara, G. (2007) How fish do geometry in large and in small spaces. Animal Cognition, 10, 47-54. doi:10.1007/s10071-006-0029-4

[38] Collett, T.S., Cartwright, B.A. and Smith, B.A. (1986) Landmark learning and visuo-spatial memories in gerbils. Journal of Comparative Physiology A, 158, 835-851. doi:10.1007/BF01324825

[39] MacDonald, S.E., Spetch, M.L., Kelly, D.M. and Cheng, K. (2004) Strategies in landmark use by children, adults, and marmoset monkeys. Learning and Motivation, 35, 322-347. doi:10.1016/j.lmot.2004.03.002

[40] Spetch, M.L., Cheng, K. and MacDonald, S.E. (1996) Learning the configuration of a landmark array: I. Touchscreen studies with pigeons and humans. Journal of Comparative Psychology, 110, 55-68. doi:10.1037/0735-7036.110.1.55

[41] Spetch, M.L., Cheng, K., MacDonald, S.E., Linkenhoker, B.A., Kelly, D.M. and Doerkson, S.R. (1997) Use of landmark configuration in pigeons and humans: II. Generality across search tasks. Journal of Comparative Psychology, 111, 14-24. doi:10.1037/0735-7036.111.1.14 